\title{
Value of Multiple Computed Tomography Criteria for Prediction of Malignancy in Patients with Ovarian Mass
}

\author{
Bilge OZTOPRAK ${ }^{1}$, Savas KARAKUS ${ }^{2}$
}

Sivas, Turkey

OBJECTIVE: Computed tomography can be used as a safe, accurate and noninvasive technique for the prediction of ovarian malignancy with several criteria. We aimed to determine the sensitivity and specificity of presence of multiple computed tomography malignancy criteria for the prediction of ovarian malignancy in patients with ovarian mass.

STUDY DESIGN: From a total of 734 patients diagnosed with an adnexal mass, ovarian mass was determined in 91 contrast-enhanced abdominopelvic computed tomography images were examined for the presence of tumoral diameter (>50 mm), thick septa, wall thickness, solid component, contrast involvement, invasion, ascites, and bilaterality. The ratios of these parameters and the value of their combined use for the prediction of ovarian malignancy was assessed.

RESULTS: Of the 91 patients included in the study, in patients with benign [in 66 (72.5\%) patients] and malign [(in 25 (27.5\%) patients] ovarian mass, the mean (range) ages were 43.5 (17-82) and 58.0 (3488) years, respectively. A statistically significant relationship was determined between ovarian malignancy and all the computed tomography criteria $(p<0.05)$ except the tumoral diameter $(>50 \mathrm{~mm})$ and wall thickness $(p>0.05)$. The ROC analysis revealed that with the presence of 3 or more criteria among the 8 computed tomography criteria, the ovarian mass can be predicted as malignant at least with a sensitivity of $76 \%$ and specificity of $70 \%$.

CONCLUSION: The presence of 3 or more parameters among the 8 selected computed tomography criteria, the ovarian malignancy can be predicted at least with a sensitivity of $76 \%$ and specificity of $70 \%$ in patients with ovarian mass.

Keywords: Computed tomography, Histopathology, Morphological characteristics, Ovarian cancer

Gynecol Obstet Reprod Med 2018;24(1):42-46

\section{Introduction}

Adnexal masses are associated with functional, congenital, inflammatory and neoplastic processes in the adnexa and adjacent organs. The majority are of ovarian origin and benign. In $5 \%-10 \%$ of the female population surgery is applied for suspicion of an ovarian neoplasm at some point in the lifetime. Malignancy is determined in $13 \%-21 \%$ of these women $(1,2)$.

Early identification of ovarian cancer and early surgical in-

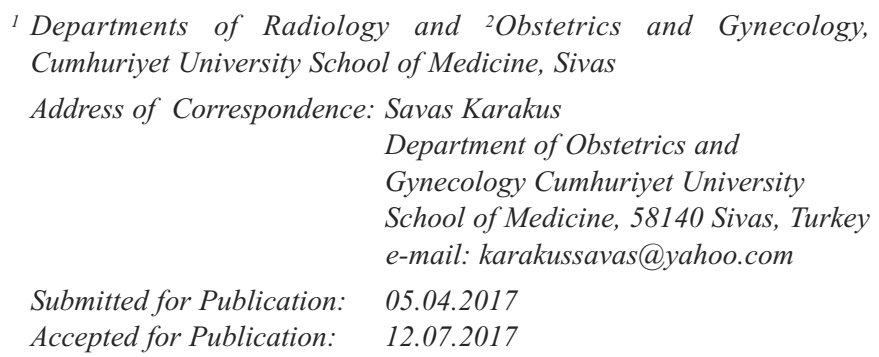

\begin{tabular}{|c|c|}
\hline \multicolumn{2}{|c|}{ Access this article online } \\
\hline 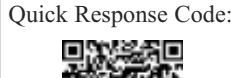 & $\begin{array}{l}\text { Website: www.gorm.com.tr } \\
\text { e- mail: info@gorm.com.tr }\end{array}$ \\
\hline 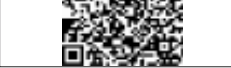 & DOI:10.21613/GORM.2016.706 \\
\hline
\end{tabular}

How to cite this article: Oztoprak B. and Karakus S. Value of Multiple Computed Tomography Criteria for Prediction of Malignancy in Patients with Ovarian Mass. Gynecol Obstet Reprod Med 2017;23(1):00-00 tervention have been shown to prolong the life of the patient (3). In contrast to other gynecological cancers, ovarian tumors are generally diagnosed at an advanced stage as there are no specific symptoms (4). Therefore, early diagnosis and treatment of an ovarian tumor is very important. For the differentiation of ovarian masses and the decision to apply surgery, the diagnostic methods to be used include physical examination, biochemical markers (5), ultrasonography (US) (6,7), magnetic resonance imaging (MRI) (8), positron emission tomography (PET) (9) and computed tomography $(\mathrm{CT})(8,9)$. In previous studies, it has been reported that MRI is superior to CT and US in the differential diagnosis of ovarian masses but that there was no difference between these methods in malignantbenign differentiation (10). Despite US scoring in malignantbenign differentiation, incorrect diagnoses can be made (11). Some criteria are used in the malignant-benign differentiation of pathologies determined radiologically. The criteria which suggest a malignant ovarian mass on $\mathrm{CT}$ are a large size, presence of thick septa $(>3 \mathrm{~mm})$, a thick wall, solid component, papillary projections, contrast enhancement, ascites or invasion and being bilateral (12).

Differential diagnosis of adnexal masses is challenging $(13,14)$ and a definitive diagnosis is not possible without histopathological examination in the vast majority of cases, 
and still, clinical and imaging findings guide physicians for proper patient selection for surgery. The aim of this study was to determine the sensitivity and specificity of presence of multiple CT malignancy criteria in patients with ovarian mass.

\section{Material and Method}

After the approval of the study protocol by the Human Research Ethics Committee of our university, in this crosssectional study, the abdominopelvic CT findings and patient records of 734 patients operated on for a pelvic mass in our hospital from September 2013 to February 2016 were examined. Of these, 629 patients were excluded from the study because of extra-ovarian pathology (endometrium carcinoma, leiomyoma, leiomyosarcoma, etc.). Fourteen of 105 patients with ovarian mass were excluded because of a lack of abdominopelvic CT recorded on PACS, absence of a contrastenhanced CT, or because the CT images were not suitable for optimum evaluation due to the presence of artifacts (motion artifacts, metallic artifacts, beam-hardening, etc.). A 64x2 multidetector computed tomography device (Aquilion CX, Toshiba, Tokyo, Japan) was used to obtain the CT images after intravenous administration of a $1 \mathrm{~mL} / \mathrm{kg}$ iodinated contrast material. Thus, a total of 91 patients determined with an ovarian mass were included in the study. The presence of CT criteria for the prediction of ovarian malignancy, including presence of tumoral diameter $(>50 \mathrm{~mm})$, thick septa, wall thickness, solid component, contrast involvement, invasion, ascites, and bilaterality were recorded.

\section{Statistical analysis}

Statistical analyses were performed with IBM SPSS version 22 (IBM Corporation, Armonk, NY, USA). Data were presented as median (min-max) or percentage (\%) as appropriate. Correlations between the presence of malignancy and CT criteria were analyzed with chi-square test. To determine the cut-off for the number of presence of multiple CT criteria in combination to predict malignant ovarian cancer, receiveroperating curve (ROC) analyses were carried out. A value of $\mathrm{p}<0.05$ was accepted as statistically significant.

\section{Results}

In the total 91 patients included in the study, the mean (range) ages were 43 (17-82) and 58 (34-88) years in patients with benign and malign ovarian tumor, respectively. Table 1 shows the presence of CT criteria for the prediction of ovarian malignancy, including presence of tumoral diameter $(>50$ $\mathrm{mm}$ ), thick septa, wall thickness, solid component, contrast involvement, invasion, ascites, and bilaterality in patients with benign and malignant ovarian tumor. The ratio of presence of thick septa, solid component, contrast involvement, invasion, ascites, and bilaterality were significantly higher in patients with malignant ovarian tumor $(\mathrm{p}<0.05)$ except tumoral diameter $(>50 \mathrm{~mm})$ and wall thickness, providing no significant difference $(p>0.05)$.

Histopathologically, 25 (27.5\%) of the patients were diagnosed with a malignant mass, and $66(72.5 \%)$ with a benign mass (Figures 1-4).

After multiple ROC analyses with the combination of CT criteria from 2 to 8 , ROC analysis revealed that the presence of 3 or more computed tomography criteria provided more optimal sensitivity and specificity for the prediction of malignancy in patients with ovarian tumors. The ROC analysis revealed that with the presence of 3 or more criteria among the $8 \mathrm{CT}$ criteria, the ovarian mass can be predicted as malignant at least with a sensitivity of $76 \%$ and specificity of $70 \%$ (Figure 5).

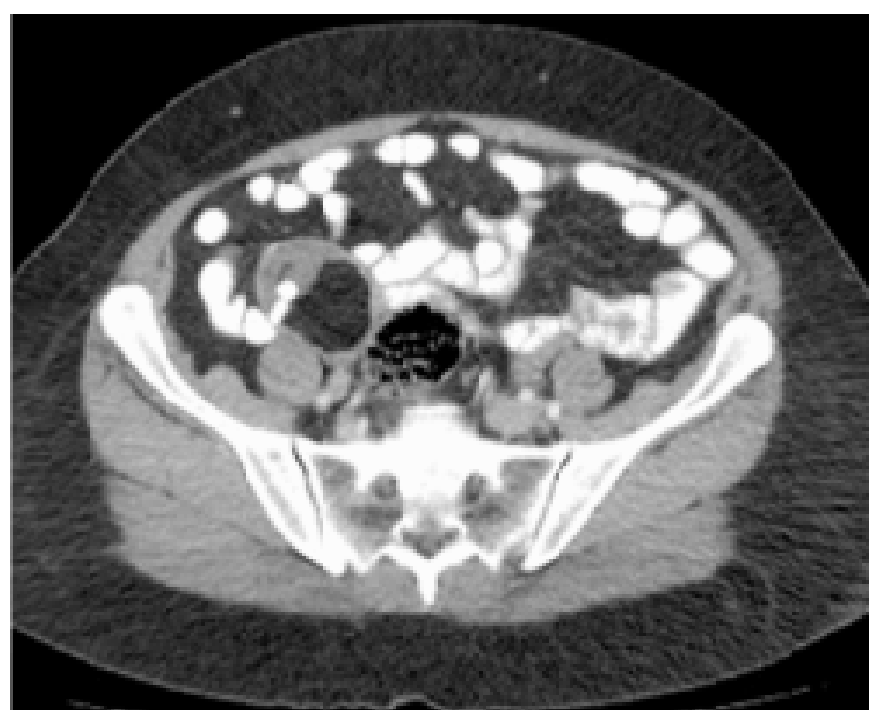

Figure 1: Typical mature cystic teratoma ovarian mass containing calcified structures and fat tissue on the right.

Table 1: Computed tomography criteria for the prediction of ovarian malignancy, including presence of tumoral diameter (>50 mm), thick septa, wall thickness, solid component, contrast involvement, invasion, ascites, and bilaterality.

\begin{tabular}{llll}
\hline Presence & Benign $(\mathrm{n}=66)$ & Malign $(\mathrm{n}=25)$ & Significance \\
\hline Tumoral diameter $(>50 \mathrm{~mm})$ & $54(81.8 \%)$ & $20(80 \%)$ & $\mathrm{NS}$ \\
Thick septa & $17(25.8 \%)$ & $17(25.8 \%)$ & $\mathrm{p}<0.05$ \\
Wall thickness & $16(24.2 \%)$ & $5(20.0 \%)$ & $\mathrm{NS}$ \\
Solid component & $18(27.3 \%)$ & $19(76.0 \%)$ & $\mathrm{p}<0.05$ \\
Contrast involvement & $36(54.5 \%)$ & $22(88.0 \%)$ & $\mathrm{p}<0.05$ \\
Invasion & $7(10.6 \%)$ & $9(36.0 \%)$ & $\mathrm{p}<0.05$ \\
Ascites & $8(12.1 \%)$ & $12(48.0 \%)$ & $\mathrm{p}<0.05$ \\
Bilaterality & $13(19.7 \%)$ & $11(44 \%)$ & $\mathrm{p}<0.05$ \\
\hline
\end{tabular}




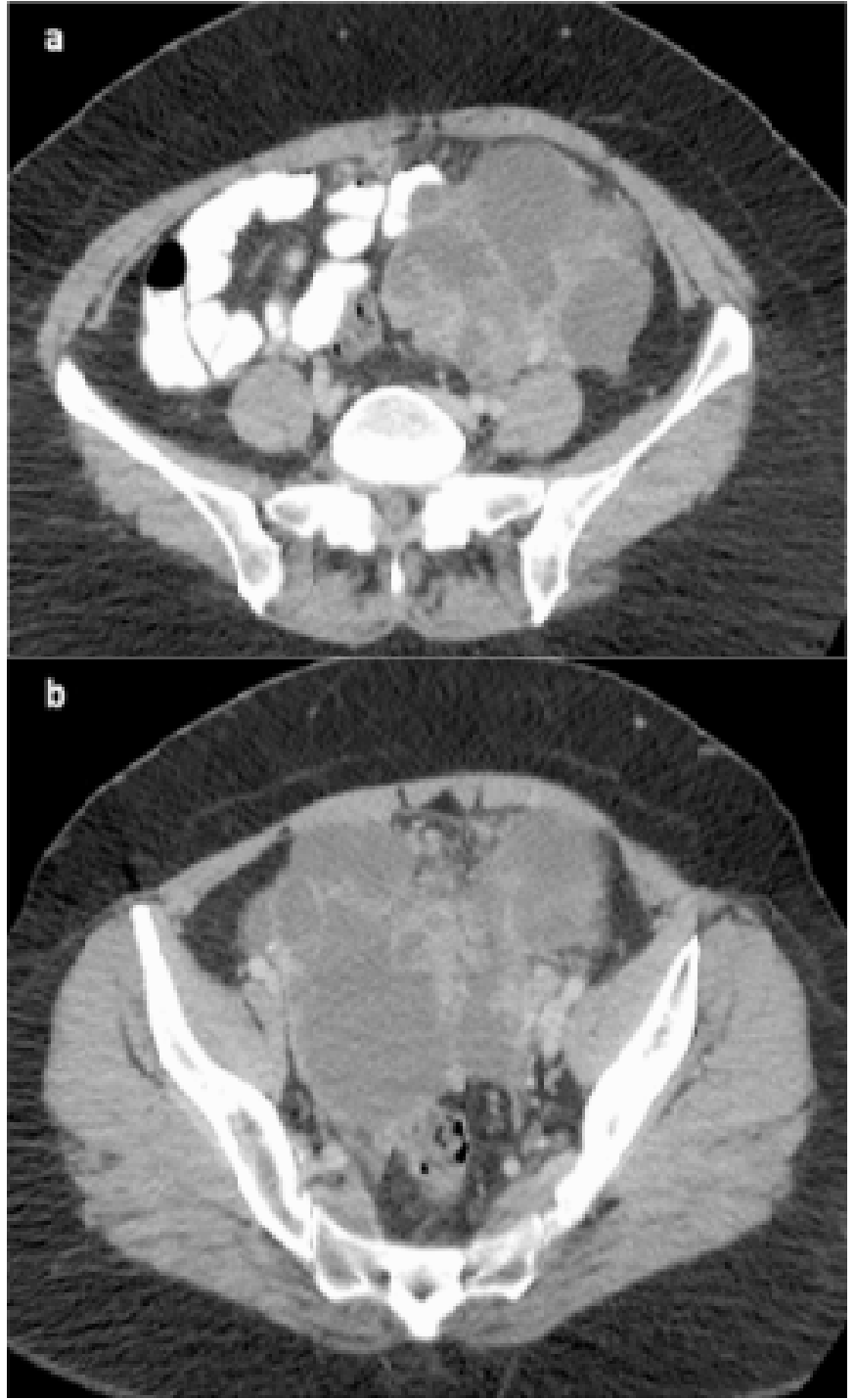

Figure 2. Contrast involvement of thick septae and solid components, large size and bilaterality observed, suggesting malignancy in ovarian masses histopathologically confirmed as bilateral serous cystadenocarcinoma.

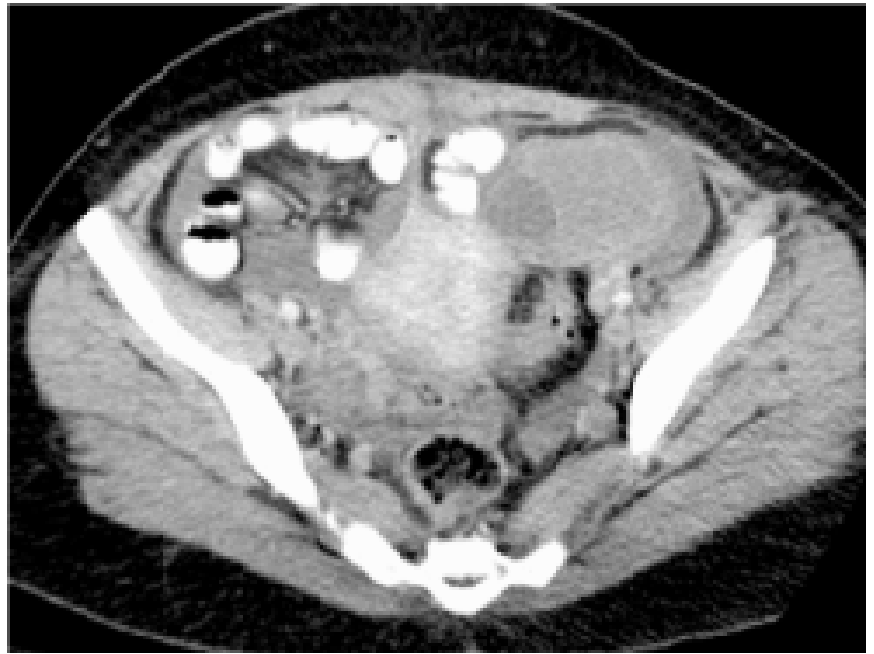

Figure 3. Ovarian mass with thick inner wall contrast involvement containing cystic components of 2 different densities. The lesion was histopathologically diagnosed as endometriosis.
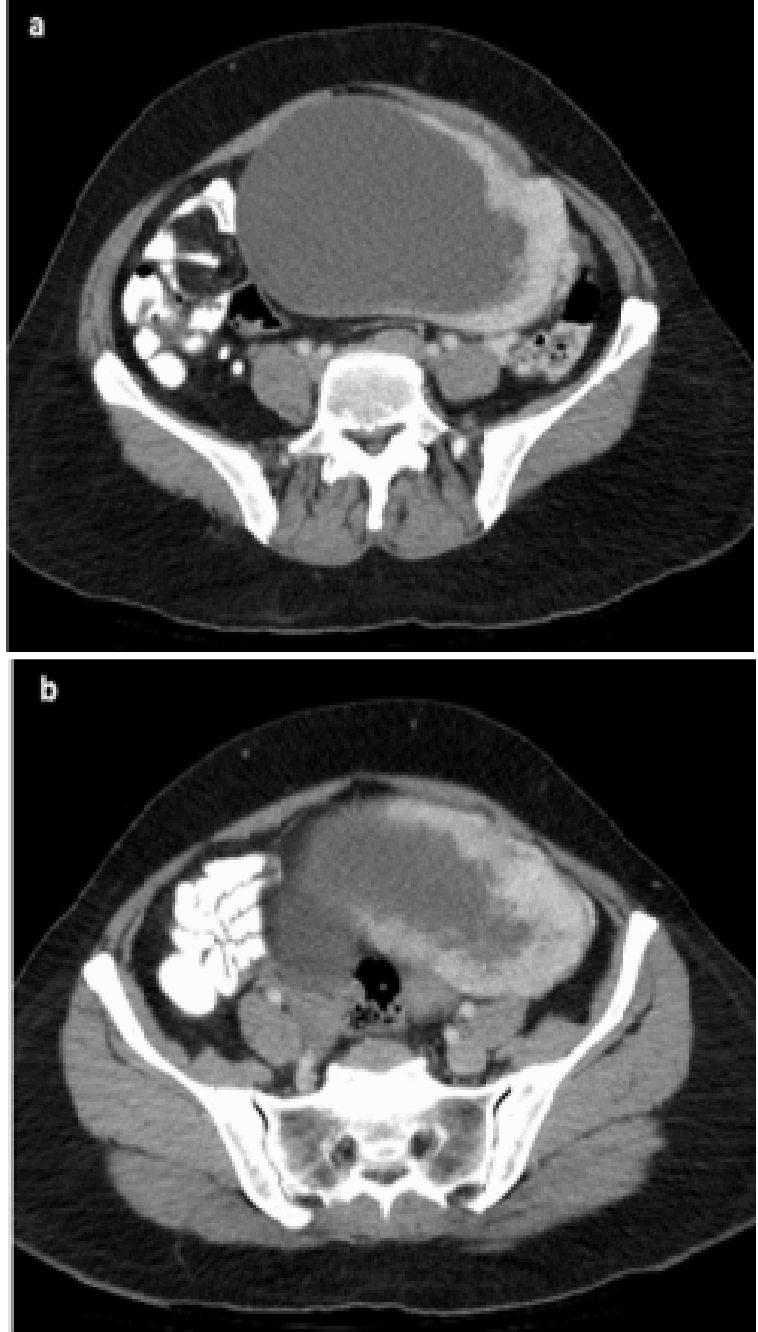

Figure 4. Large cystic mass with thick, irregular inner walls with contrast involvement diagnosed as benign left-side ovarian fibroma.

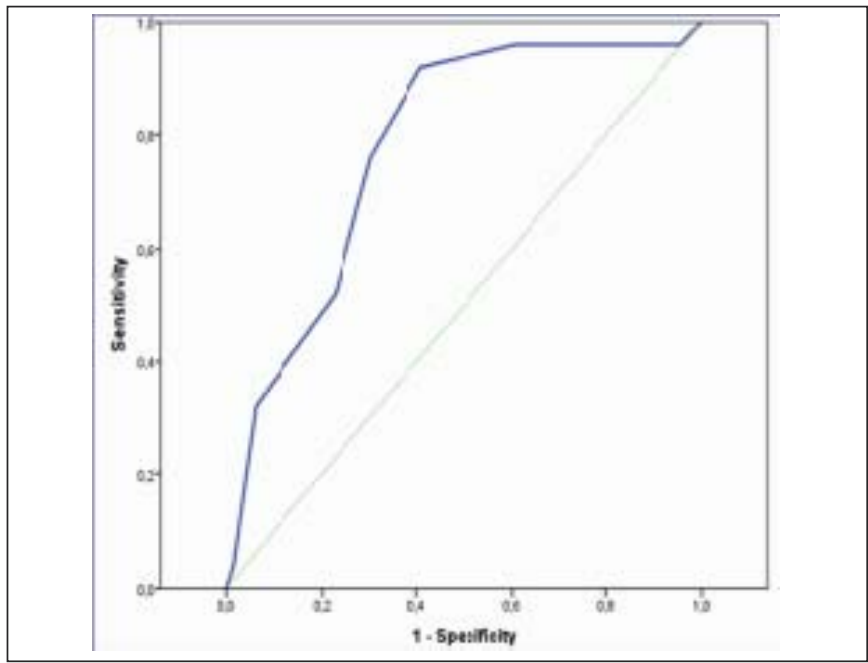

Figure 5. Receiver-operating characteristic curve analysis revealed that the presence of 3 or more computed tomography criteria provided more optimal sensitivity and specificity for the prediction of malignancy in patients with ovarian tumors. The presence of 3 or more computed tomography criteria can predict malignant ovarian cancer at least with a sensitivity of $76 \%$ and specificity of $70 \%$. 


\section{Discussion}

The results of this study showed that the mass size and wall thickness did not give reliable information in respect of histopathological diagnosis. However, a relationship was determined between malignancy and thick septae, solid components, invasion, ascites and bilateral involvement of the ovaries. Of these, the relationships of solid components and ascites with malignancy were seen to be more significant than the other parameters. When interpreting CT images, these findings can be very helpful in making a decision in favor of malignancy. Nevertheless, although the presence of solid components in ovarian lesions on all radiological imaging methods is an important criterion suggesting malignancy, it could be a misleading finding in benign fibromas, as was seen in this study. Although these types of ovarian masses are benign, a suspicion of malignancy may occur because of the presence of solid components. Instead of use of single-criterion, we tried to find optimal combination of $\mathrm{CT}$ criteria for the prediction of ovarian malignancy, and after ROC analysis revealed that the presence of 3 or more criteria among the $8 \mathrm{CT}$ criteria can predict ovarian malignancy at least with a sensitivity of $76 \%$ and specificity of $70 \%$ in patients with ovarian mass.

After USG, CT is the most commonly used imaging method for imaging the female genital system. It provides significant clues in the characterization of the mass. Particularly in the diagnosis of mature cystic teratoma, $\mathrm{CT}$ is superior to ultrasound and diagnosis is made with a very high degree of accuracy from the good visualization of the fat and calcified structure content. However, in the absence of mesodermal and ectodermal components, teratomas are confused with other ovarian masses and it is not possible to make a specific diagnosis with imaging methods. However, because of an expensive and less readily available compared to USG, CT is not an appropriate imaging method for the choice for the differentiation of adnexal masses and for the decision whether or not to proceed with surgery.

Computed tomography is the most commonly used imaging technique in the differentiation of adnexal masses. Various studies have used CT to distinguish benign or malignant adnexal masses (15-18). Shen-Gunther et al. (15) investigated the relationship between the presence of ascites and presence of ovarian malignancy in CT and the relationship between the presence of ascites and the histological type of ovarian tumor (benign, borderline or malignant) they found 57 benign tumors, only 5 patients $(9 \%)$ had fewer peritoneal effusions. Borderline was found in 7 (58\%) of 12 patients. Of the 56 malignant tumors, $41(73 \%)$ had ascites. They found that; because half of the borderline over tumors and early stage ovarian tumors do not form an ascites, the absence of ascites in the absence of a benign or malignant cannot be made clear. However, the presence of ascites is highly predictive of ovarian malignancy.
Zhang et al. (16) demonstrated that CT can be used to characterize adnexal masses. They used two different radiological interpretations to evaluate adnexal masses preoperatively and postoperative histopathological findings and that adnexal malignancies use CT imaging to characterize adnexal masses. They confirmed the diagnostic value of contrast-enhanced computerized tomography by this way. They found that contrast-enhanced helix BT was more efficient than identifying adnexal masses as malignant because of the increased involvement of contrast media, and that the need for additional imaging studies could possibly be eliminated before treatment selection.

Santoso et al. (17) evaluated the presence of ascites, solid component, and metastasis by evaluating preoperative CT with 324 adnexal masses diagnosed with adnexal masses in 324 adenocarcinoma patients in order to determine whether adnexal masses were malignant. In the malignant patient group, the presence of solid component, metastasis and ascites were significantly higher. They have concluded that CT could be used to identify possible malignant risks of adnexal masses.

Tsili et al. (18) conducted a multidetector computed tomography (MDCT) on a 16-row CT scanner for the detection and differentiation of adnexal masses, the thickness of septae, the presence of cystic solid mass, ascites presence, and invasion. They compared these findings with those of malignancies which could be identified with post-operative histopathologically. According to CT findings, they found a relationship with the bilaterality, cystic solid mass, septae thickening, and ascites with malignancy. However, they found more significant relationship between invasions and malignancy.

As summarized above from the pertinent literature, there are many studies assessing the value CT criteria for the prediction of ovarian malignancy and although they noted the value of various CT criteria for this purpose, there is no consensus on the combined use of CT criteria. According to our findings the presence of at least 3 criteria in combination can predict ovarian malignancy more reliably.

The ability to make malignant-benign differentiation of ovarian masses in the preoperative period remains a problem which has not yet been solved. As existing diagnostic methods remain insufficient, most of the time diagnosis can only be made during the operation or histopathologically afterwards. As has been demonstrated in this study, despite the definitive histopathological diagnosis of ovarian masses, the morphological properties seen on CT are of importance in preoperative diagnosis. Further prospective studies of the correlation between surgery, radiology and pathology will make further contributions of radiologists to the surgical planning.

The main limitation of this study was its cross-sectional design, therefore, it was not possible to consider additional pathologies and problems encountered during surgery. Only 
the morphological properties of the ovarian mass were taken into consideration and there was no evaluation of secondary findings including distant organ involvement and laboratory findings. The use of ROC analysis during the assessment of value of combined criteria for the prediction of ovarian malignancy with CT findings in patients with ovarian mass was the strength of this study, since there was not enough study performed with this purpose.

In conclusion, when there are 3 or more of the $\mathrm{CT}$ criteria among the $8 \mathrm{CT}$ criteria, including the presence of tumoral diameter (>50 mm), thick septa, wall thickness, solid component, contrast involvement, invasion, ascites, and bilaterality, can predict ovarian malignancy at least with a sensitivity of $76 \%$ and specificity of $70 \%$ in patients with ovarian mass.

: The authors declare that there are no conflicts of interest.

\section{References}

1. DiSaia PJ. The Adnexal Mass and Early Ovarian Cancer. In: DiSaia PJ, Creasman WT, Editors. Clinical Gynecologic Oncology. Amsterdam: Elsevier; $7^{\text {th }}$ Edition. 2007;283-312.

2. Howlader N, Noone AM, Krapcho M, Miller D, Bishop K, Kosary CL, Yu M, Ruhl J, Tatalovich Z, Mariotto A, Lewis DR, Chen HS, Feuer EJ, Cronin KA (eds). SEER Cancer Statistics Review, 1975-2014, National Cancer Institute. Bethesda, MD, https://seer.cancer.gov/csr/ 1975_2014/.

3. Lajtman E, Mlyncek M, Uharcek P, Matejka M. Followup after primary therapy of malignant epithelial ovarian tumors. Bratisl Lek Listy 2011;112(10):579-85.

4. Jelovac D, Armstrong DK. Recent progress in the diagnosis and treatment of ovarian cancer. CA Cancer J Clin 2011;61(3):183-203.

5. Coleman RL, Herzog TJ, Chan DW, Munroe DG, Pappas TC, Smith A, et al. Validation of a second-generation multivariate index assay for malignancy risk of adnexal masses. Am J Obstet Gynecol 2016;215(1):82. e1-82. e11.

6. Ersoy E, Tokmak A, Ersoy AO, Evliyaoglu O. Preoperative Differentiation Between Malignant and Benign Ovarian Masses in Patients with Normal CA-125 Levels. Gynecol Obstet Reprod Med. 2016;22(2):94-98

7. Erdemoglu E, Gunyeli I, Kamac1 M, Mungan T. Adnexial Mass in the Adolescents. Gynecol Obstet Reprod Med. 2011;17(1):39-41
8. Jung SE, Lee JM, Rha SE, Byun JY, Jung JI, Hahn ST. CT and MR imaging of ovarian tumors with emphasis on differential diagnosis. Radiographics. 2002;22(6):1305-25.

9. Oldan JD, Patel PS. Positron Emission Tomography/ Computed Tomography for Gynecologic Malignancies. Obstet Gynecol Surv. 2016;71(9):545-56.

10. Kurtz AB, Tsimikas JV, Tempany CM, Hamper UM, Arger $\mathrm{PH}$, Bree RL. Diagnosis and staging of ovarian cancer: comparative values of Doppler and conventional US, CT, and MR imaging correlated with surgery and histopathologic analysis: report of the Radiology Diagnostic Oncology Group. Radiology 1999; 212(1):19-27.

11. Sassone AM, Timor-Tritsch IE, Artner A, Westhoff C, Warren WB. Transvaginal sonographic characterization of ovarian disease: evaluation of a new scoring system to predict ovarian malignancy. Obstet Gynecol 1991; 78(1):70-6.

12. Grabowska-Derlatka L, Derlatka P, Palczewski P, DanskaBidzinska A, Pacho R. Differentiation of ovarian cancers from borderline ovarian tumors on the basis of evaluation of tumor vascularity in multi-row detector computed tomography--comparison with histopathology. Int $\mathrm{J}$ Gynecol Cancer 2013;23(9):1597-602.

13. Suh-Burgmann E, Kinney W. Potential harms outweigh benefits of indefinite monitoring of stable adnexal masses. Am J Obstet Gynecol 2015;213(6): 816.e1-4.

14. Suh-Burgmann E, Kinney W. The Value of Ultrasound Monitoring of Adnexal Masses for Early Detection of Ovarian Cancer. Front Oncol 2016; 6:25

15. Shen-Gunther J, Mannel RS. Ascites as a predictor of ovarian malignancy. Gynecol Oncol. 2002;87(1):77-83.

16. Zhang J, Mironov S, Hricak H, Ishill NM, Moskowitz CS, Soslow RA, Chi DS. Characterization of adnexal masses using feature analysis at contrast-enhanced helical computed tomography. J Comput Assist Tomogr 2008;32 (4):533-40.

17. Santoso JT, Robinson A, Suganda S, Praservit S, Wan JY, Ueland F. Computed tomography adnexal mass score to estimate risk for ovarian cancer. Arch Gynecol Obstet 2014;289(3):595-600.

18. Tsili AC, Tsampoulas C, Charisiadi A, Kalef-Ezra J, Dousias V, Paraskevaidis E, Efremidis SC. Adnexal masses: accuracy of detection and differentiation with multidetector computed tomography. Gynecol Oncol 2008;110(1):22-31. 